\title{
Highly Hydrophilic Luminescent Magnetic Mesoporous Carbon Nanospheres for Control Release of Anticancer Drug and Multimodal Imaging
}

Sasmita Mohapatra, ${ }^{*}$ Smruti R. Rout ${ }^{\dagger}$ Rahul K. Das, ${ }^{\dagger}$ Santoshi Nayak, ${ }^{+}$and Sudip K. Ghosh ${ }^{\ddagger}$

Electronic Supplementary Information

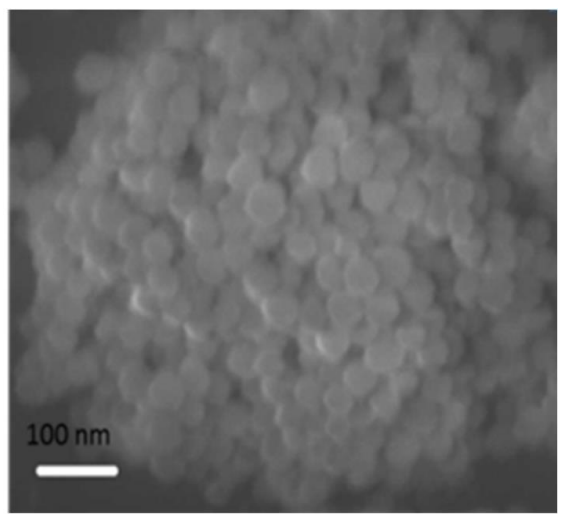

Figure S1 SEM image of $\mathrm{CoFe}_{2} \mathrm{O}_{4} @ \mathrm{mSiO}_{2}$
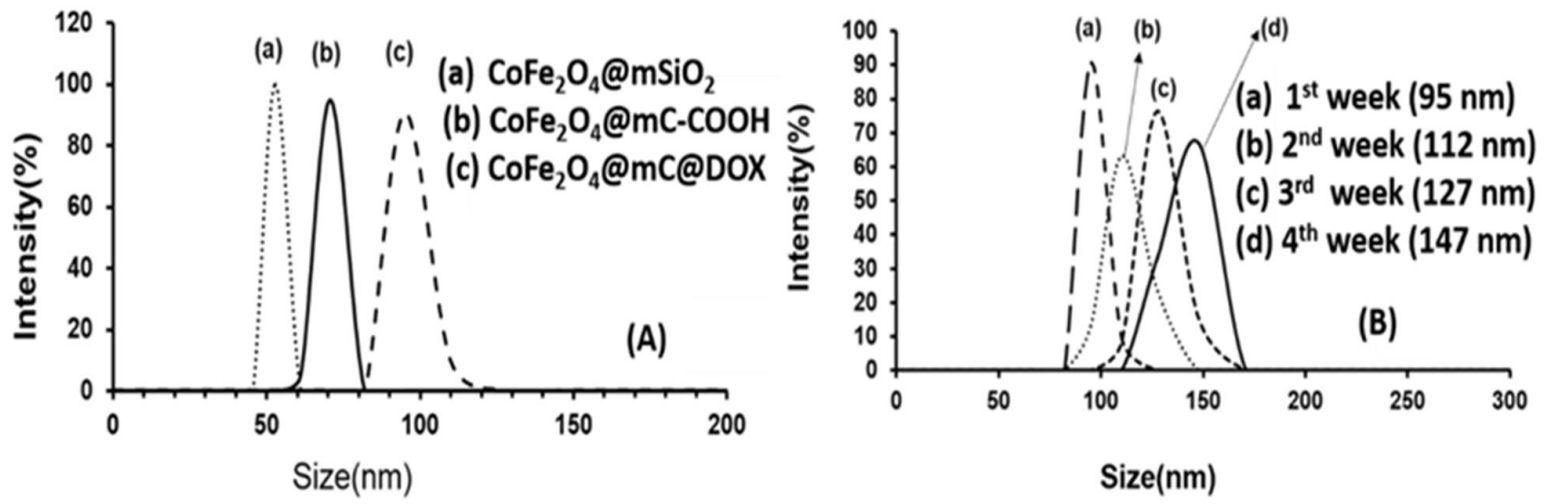

Figure S2 (a) Particle size distribution at each step of modification (b) Stability of DOX@CoFe $\mathrm{O}_{4} @ \mathrm{mC}$ after several weeks. 

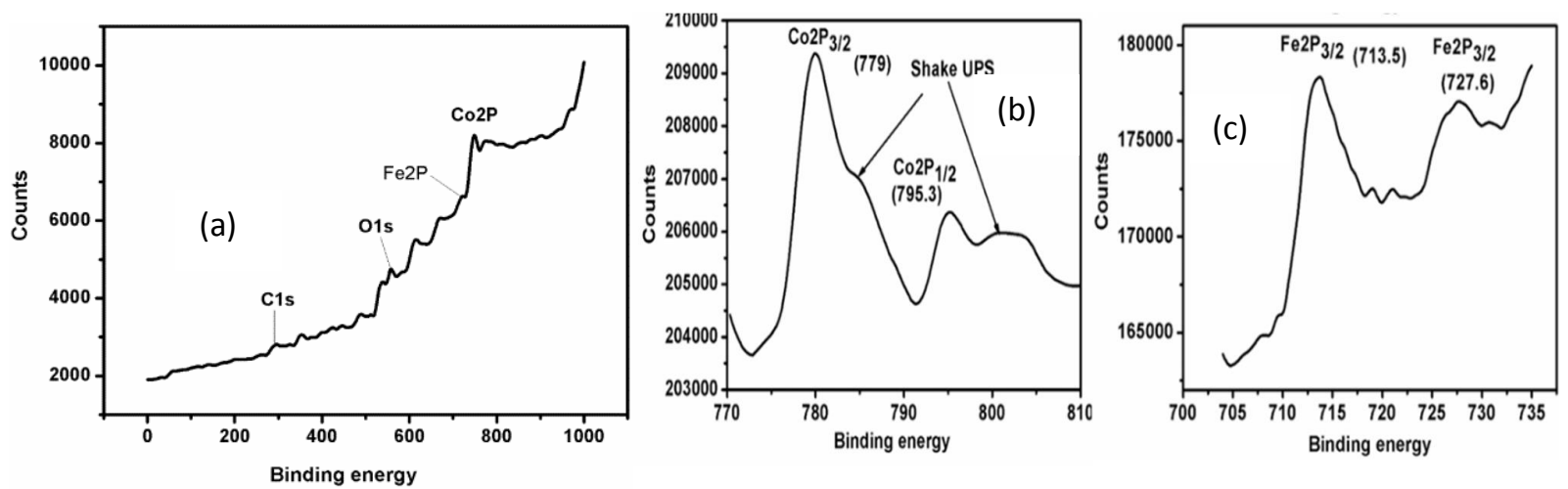

Figure S3 (a) XPS survey spectrum of $\mathrm{CoFe}_{2} \mathrm{O}_{4} @ \mathrm{mC}$, (b) High resolution scan of Co2p, (c) Fe2p regions.

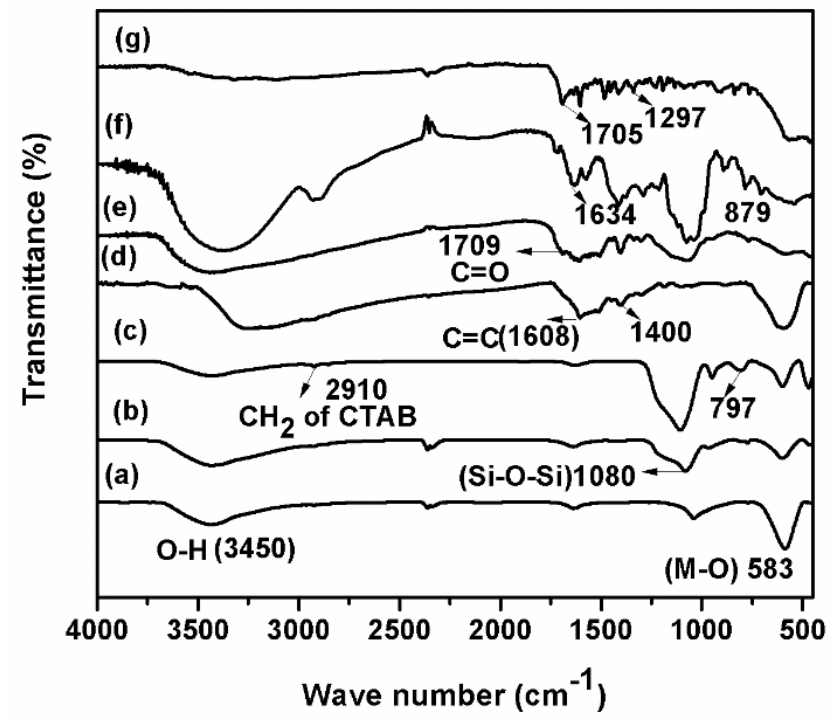

Figure S4 FTIR spectra of (a) $\mathrm{CoFe}_{2} \mathrm{O}_{4}$, (b) $\mathrm{CoFe}_{2} \mathrm{O}_{4} @ \mathrm{mSiO}_{2}$, (c) $\mathrm{CoFe}_{2} \mathrm{O}_{4} @ \mathrm{SiO}_{2} / \mathrm{CTAB}$, (d) $\mathrm{CoFe}_{2} \mathrm{O}_{4} @ \mathrm{mC}$, (e) $\mathrm{CoFe}_{2} \mathrm{O}_{4} @ \mathrm{mC} @ \mathrm{COOH}$, (f) DOX and (g) $\mathrm{CoFe}_{2} \mathrm{O}_{4} @ \mathrm{mC} @ \mathrm{DOX}$ 

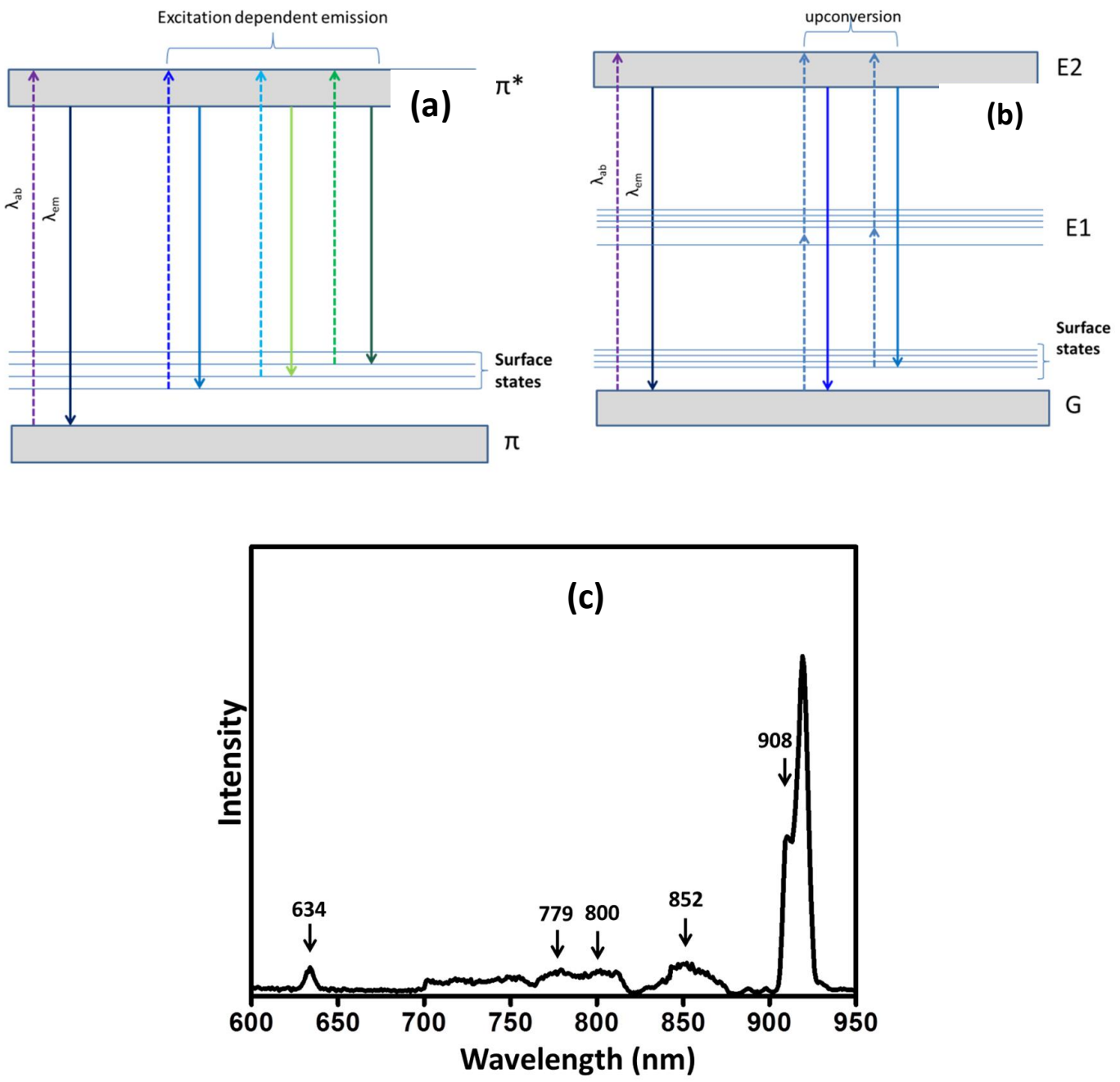

Figure S5 Illustration showing (a) excitation dependent emission (b) upconversion luminescence in mesoporous carbon, (c) excitation spectrum recorded

1) Photoluminescence of carbon shells

The photoluminescence exhibited by the carbon shell is attributed to the 'electron transition of $\mathrm{C}=\mathrm{C}$ in the skeleton of carbon shell which is composed of cyclic aromatic hydrocarbons. The higher excitation energy of photons promotes '-electrons from ground level' to higher state '*. The emission photons are of lower energy due to the energy dissipation as shown in the figure S5a.

2) Tunable photoluminescence

As described in the experimental section, the surface of $\mathrm{CoFe}_{2} \mathrm{O}_{4} @ \mathrm{mC}$ was oxidized to $\ddot{\mathrm{I}} \mathrm{COOH}$ by heating at $300 € \mathrm{C}$ in presence of air. Now the carbon shell consists of $\mathrm{C}=\mathrm{C}$ core, 
as well as oxygen containing functional groups $(\mathrm{C}=\mathrm{O}, \mathrm{C}-\mathrm{OH}, \mathrm{C}-\mathrm{O}-\mathrm{C})$ on the surface forming different surface states which are the energy between ' and '* states of carbon shell which may result in a series of emissive traps as shown in figure below. When certain excitation wave length illuminates carbon shell a surface emissive trap will predominate emission. As the excitation wavelength changes, another corresponding emissive trap will become dominant. Thus the energy states introduced by surface functional groups play an important role in the multicolour emission of carbon shell.

\section{3) Upconversion PL}

The upconversion photoluminescence of mesoporous carbon may be attributed to the multiphoton active process, in which when excitation energy is resonant with transition from ground level $\mathrm{G}$ to excited metastable level E1, light absorption occurs. Immediately a second photon is absorbed that promotes MCNs from excited metastable level E1 to higher state E2. Thus the successive absorption of pump photons results in the emission of shorter wavelength photons compared with the excitation wavelength. The above discussion has been included in the ñsupplementary informationò
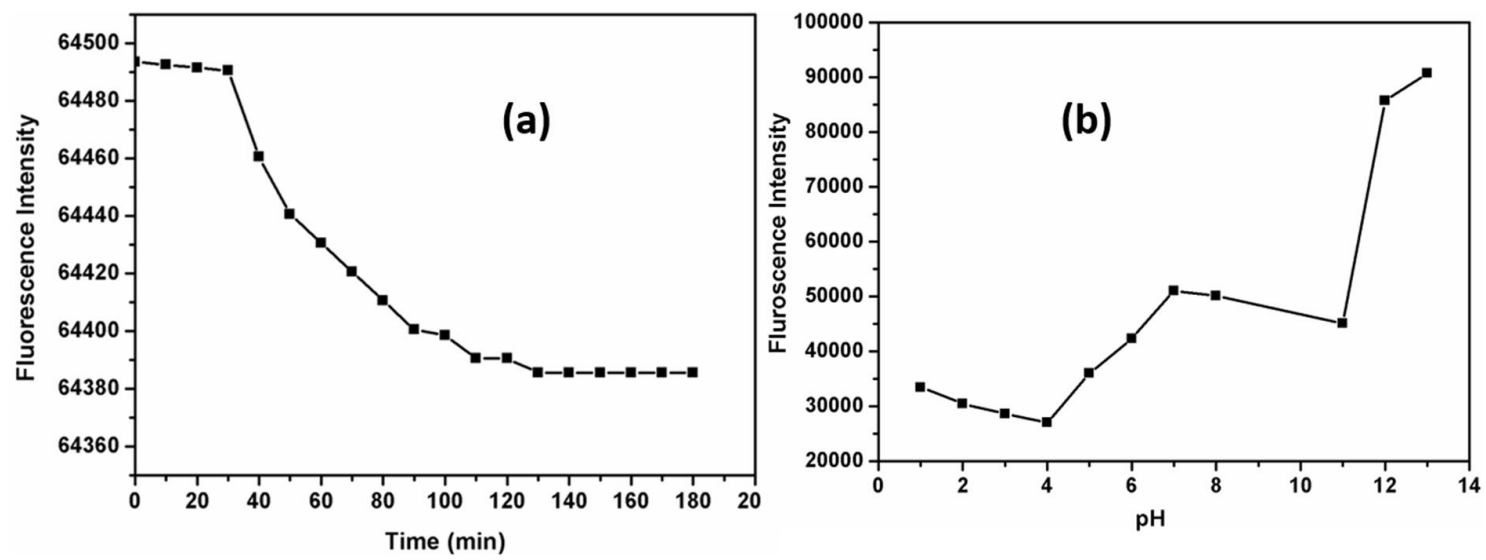

Figure S6 (a)Effect of time on fluorescence intensity of magnetic mesoporous carbon in DI water, (b) Effect of $\mathrm{pH}$ on the fluorescence intensity of magnetic mesoporous carbon. 


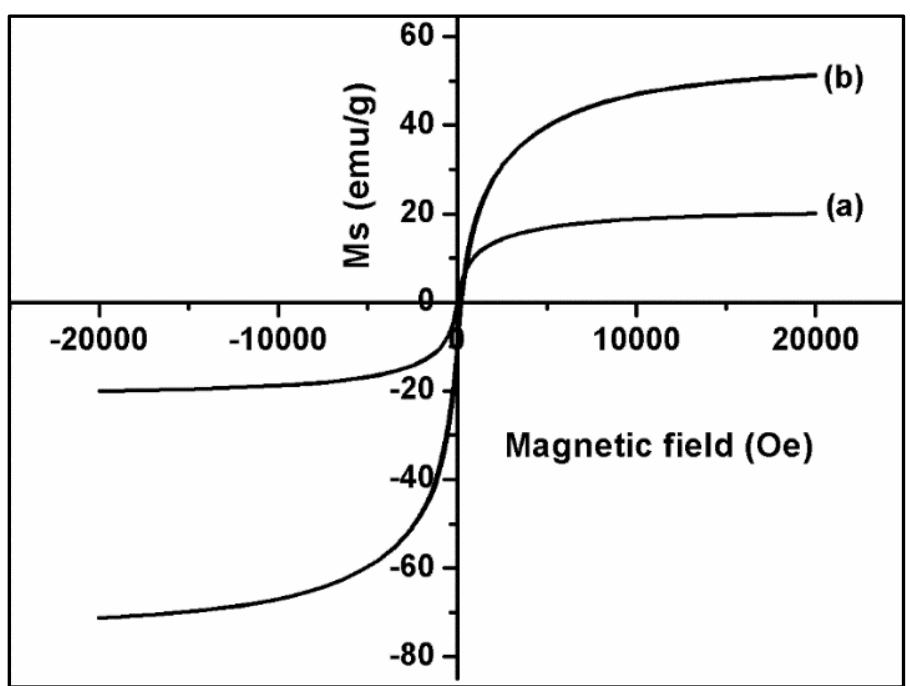

Figure S7. Magnetization curve $(\mathrm{M} \sim \mathrm{H})$ at room temperature for samples (a) $\mathrm{CoFe}_{2} \mathrm{O}_{4} @ \mathrm{mSiO}_{2} @ \mathrm{RF}$ and (b) $\mathrm{CoFe}_{2} \mathrm{O}_{4} @ \mathrm{mC}$

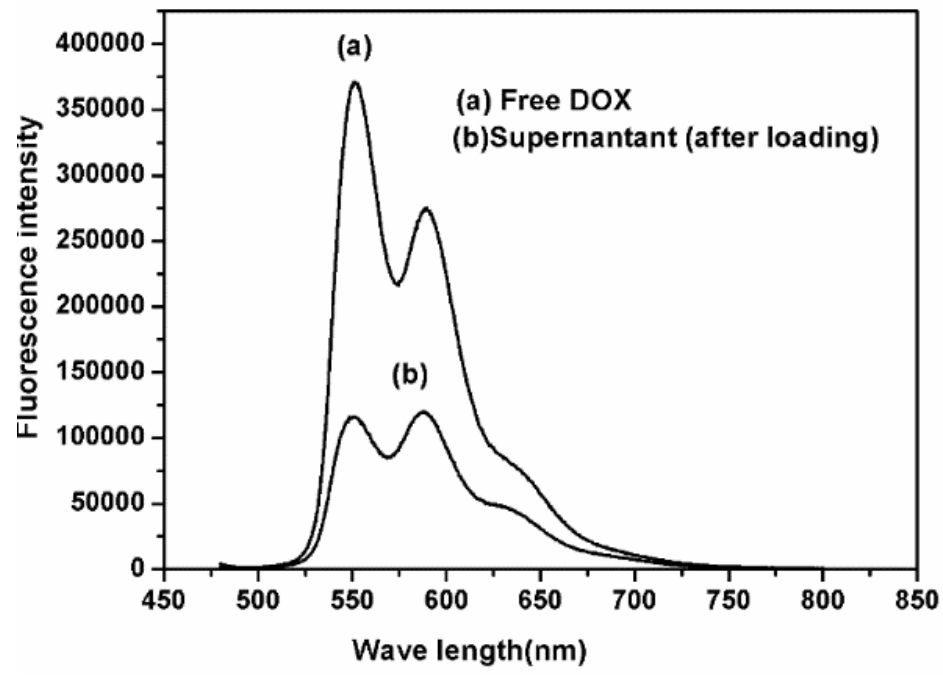

Figure S8 (a) Fluorescence spectra intensity of free DOX and (b) supernatant after loading 

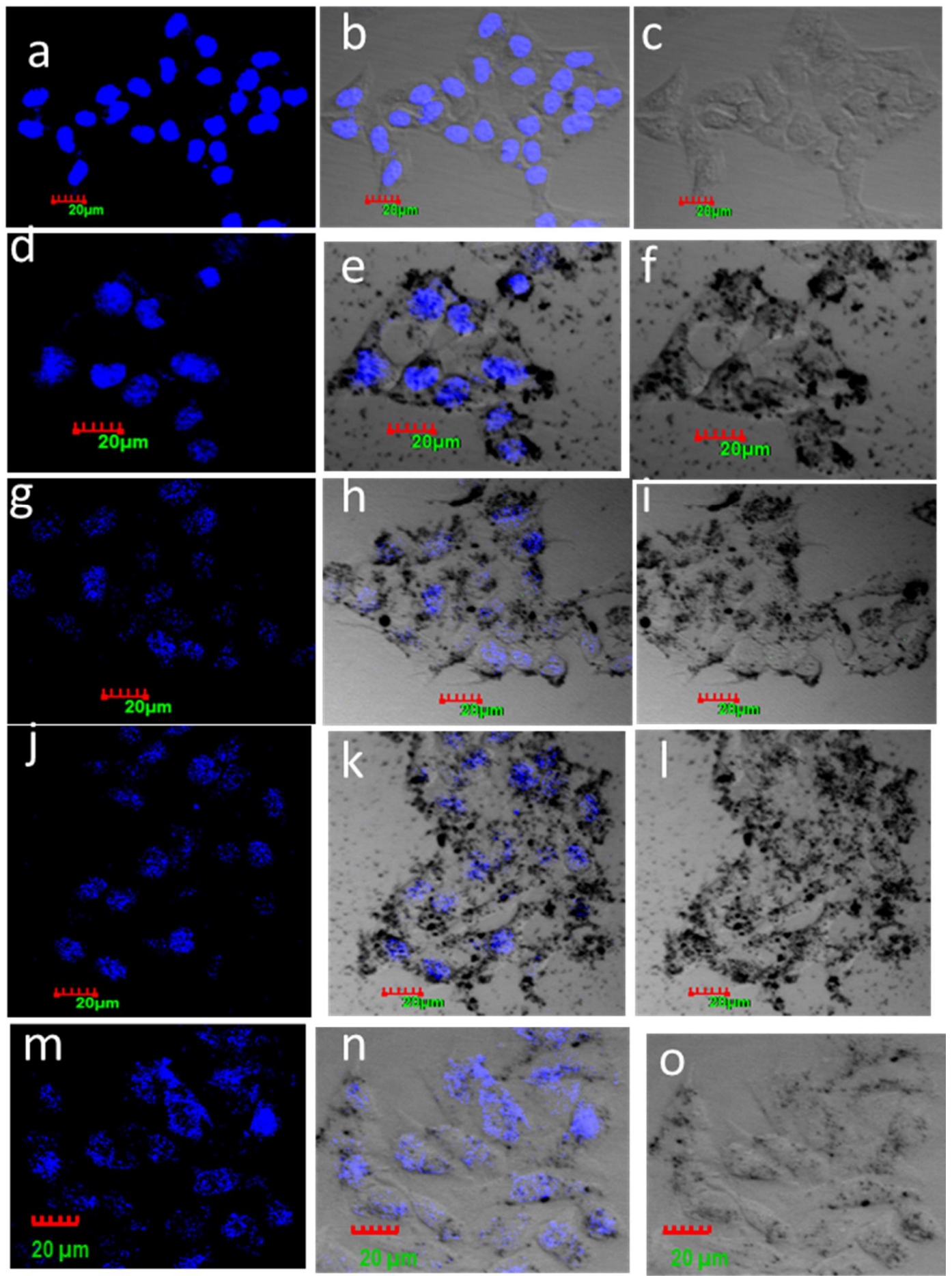

Figure S8 (a) DAPI fluorescence, bright field and merged images of HeLa cells after incubation with $2 \mu \mathrm{g} / \mathrm{ml}$ of $\mathrm{CoFe}_{2} \mathrm{O}_{4} @ \mathrm{mC} @ \mathrm{DOX}$. Images have been taken at different time 
intervals to verify cell apoptosis. (a)-(c) cell at $0 \mathrm{~h},(\mathrm{~d})-(\mathrm{f})$ at $2 \mathrm{~h},(\mathrm{~g})-(\mathrm{i})$ at $4 \mathrm{~h},(\mathrm{j})$ to (l) at $6 \mathrm{~h}$, (m)-(o) at $8 \mathrm{~h}$.
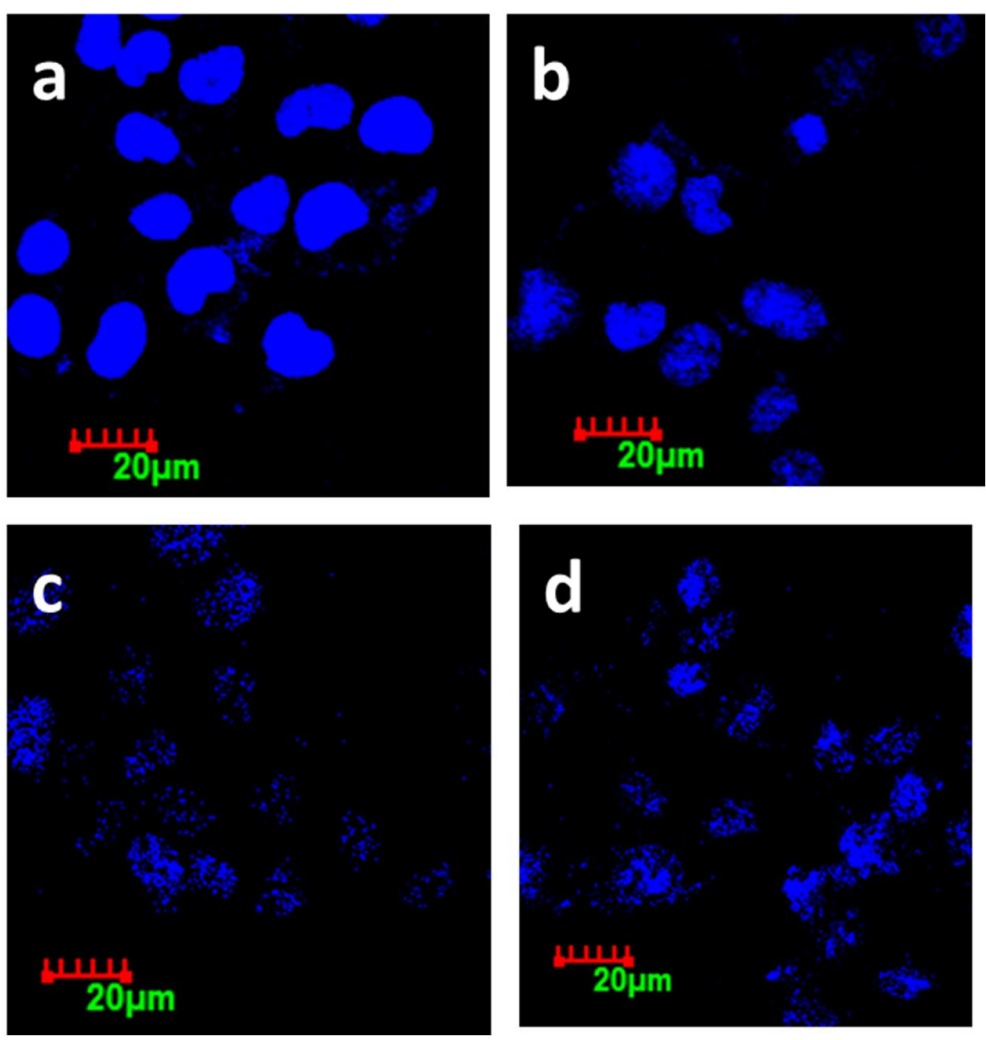

Figure S9 (A) DAPI Fluorescence image of HeLa cells, (B) after incubation $2 \mu \mathrm{g} / \mathrm{ml}$, (B) 4 $\mu \mathrm{g} / \mathrm{ml}$, (C) $5 \mu \mathrm{g} / \mathrm{ml}$ of $\mathrm{CoFe}_{2} \mathrm{O}_{4} @ \mathrm{mC} @ \mathrm{DOX}$ followed by DAPI staining which indicates that cell apoptosis increases in a dose dependent manner 


\section{Calculation of drug loading content and encapsulation efficiency}

The drug loading capacity and encapsulation efficiency was calculated as per our previously reported procedure [1]. At first the doxorubicin was adsorbed to the synthesized $\mathrm{CoFe}_{2} \mathrm{O}_{4} @ \mathrm{mC} @ \mathrm{COOH}$ particles as described in the experimental section and then particles were separated from the aqueous suspension using magnetic separator (Invitrogen). The obtained drug-loaded $\mathrm{CoFe}_{2} \mathrm{O}_{4} @ \mathrm{mC} @ \mathrm{COOH}$ nanoparticles were incubated at room temperature in overnight and were weighed. Drug concentration in supernatant was analysed by the ultraviolet absorption $\left(\gamma_{\max }=275 \mathrm{~nm}\right.$ ), with reference to a calibration curve on a UVVis spectrophotometer. The measurement were performed in triplicate. Drug-loading content and encapsulation efficiency were obtained as follows.

Drug loading content $(\%)=\longrightarrow 100$

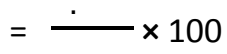

$=30.11 \%$

Encapsulation efficiency $(\%)=\longrightarrow \times 100$

$$
=\stackrel{\cdot}{-} \times 100=60 \%
$$

The drug loading content and encapsulation efficiency of DOX was found to be $30.115 \%$ and $60 \%$ respectively. 